\title{
An integrated approach for jointed rock slope stability analysis using stochastic discrete fractures: A case study in mountains area
}

\author{
Weizhong Chen ${ }^{1,3,{ }^{*}}$, Luyu Wang ${ }^{1,2}$, Xuyan Tan ${ }^{1,2}$, and Jiang Lei ${ }^{1,2}$ \\ ${ }^{1}$ State Key Laboratory of Geomechanics and Geotechnical Engineering, Institute of Rock and Soil Mechanics, Chinese Academy of \\ Sciences, Wuhan 430071, China \\ ${ }^{2}$ University of Chinese Academy of Sciences, Beijing 100049, China \\ ${ }^{3}$ Research Center of Geotechnical and Structural Engineering, Shandong University, Jinan 250100, China
}

\begin{abstract}
Stochastic discrete fractures are ubiquitous in practicing engineering and contribute much to the stability for geological mediums. In contrast to the traditional treatments based on continuum mechanics, we propose an integrated approach using discrete fractures based on an improved hybrid finite element method (FEM). A typical jointed rock slop is selected as a case study and then was simulated in the light of geological conditions for the study site. The basic equations of fracture domains are derived to generate stochastic discrete fractures which render a fundamental to the model. To implement this procedure through computer program, discrete fractures generating algorithm was developed. In the aspect of discretization, we presented a meshing scheme and proposed an algorithm to create fracture elements. To evaluate rock slope stability in a quantitative way, safety factor is calculated by strength reduction method. Numerical simulations were carried out showing the mechanical behaviors of jointed rock slope under different boundary conditions.
\end{abstract}

\section{Assement of the stability for a jointed rock slope}

\subsection{Role of the slope stablilty analysis in mountain area}

The mechanical behaviors of fractured mediums, for instance, jointed rock slope, weathered geological body and fractured reservoirs, are mainly controlled by mechanical and hydraulic properties of discrete fractures, which is one of the most typical types of discontinuous surfaces. Discontinuous in a natural geological structure include fractures, faults, cleavages and weak bedding planes, which lead to the anisotropic and homogeneous for geological mediums [1-3]. In especial, for a jointed rock slope, the influences of fractures on slope stability and mechanical response is vital important to engineering design. In the mountain area, there are a lot of infrastructure projects built on fractured geological mediums, such as the highway roadbed, bridge foundation and underground excavation. Stochastic discrete fractures are ubiquitous in this type of region and contribute much to the stability for a geological medium $[4,5]$. Apart from field investigation and experiments, it is essential developing an effective numerical approach to study the stability of fractured rocks. However, numerical simulation for a fractured medium is still a challenge topic in the field of computational mechanics $[1,6,7,8]$. In this paper, we mainly focus on the simulation approach about jointed rock slope stability.

\subsection{Traditional approaches in slope stability analysis}

In the aspect of slope stability analysis, a lot of literatures have been reported different approaches to study it [9-15]. However, it is essentially different that mechanical properties and geological structure patterns under the situations of soil and rock slope. The traditional treatment for soil slope is mainly on continuum mechanics combining with limit equilibrium method (LEM) [16,17]. In order to evaluate slope stability in a mathematical principle, Zienkiewicz introduced an index in his famous article [18], the well-known strength reduction factor (SRF), to show the stability ability for a soil slope. In the recent decades, the strength reduction method (SRM) has been successful used in practice and engineering design $[19,20]$. Nevertheless, this method is not very effective for a jointed rock slope. The main factor leading to this problem is that the effects of fractures on slope stability were neglected. To overcome the shortcoming of SRM, Zienkiewicz proposed an equivalent continuous medium model [21] to deal with it, in which he presented a novel

*Corresponding author: wzchen@whrsm.ac.cn (Weizhong Chen) 
finite element material, called jointed material, to reflect the impacts of fractures. But for a sparse fracture medium, there are still many unsolved problems for the method. On the basis of his theory, a lot of literatures have been published improved approach to simulate fractured rock slope stability [22-25], but they are all based on continuum mechanics. To simulate the influences of fracture on rock slope stability, many novel approaches were proposed, for instance, distinct element method [26,27], numerical manifold method [28], discontinuous deformation analysis [29], hybrid finite discrete method [30], mesh free analysis [31,32]. To integrate fractures into numerical model, these above methods all mainly based on an important conceptual model, discrete fracture network (DFN), which is one of the most popular treatments to deal with stochastic fractures [33-37].

\subsection{Jointed rock slope stability analysis considering the random fractures}

As mentioned above, in general, researchers studied fractured rocks through DFN. Mechanical and hydraulic characteristics of DFN are important to problems of petroleum development, fractured reservoirs, wastes disposal, hydrogeology, geothermal development $[1,6]$. Field investigation results demonstrates the distribution of fractures in a network is complex and random, and is treated usually by DFN. The concept of DFN was first introduced by Long et al. [38]. In the aspect of applications of DFN, Lei et al. [6] published a review article to state the use of DFN for geomechanical-hydrological process in fractured mediums. They emphasized that the coupled problems of DFN model is vital important to the fundamental of rock mechanics. To implement the DFN by computer programs, the corresponding computational algorithms should be developed. But there are still unsolved issues related to this topic, such as mesh generation problem, three-dimensional modeling and visualization, simulation techniques of flow and coupled process.

Following the previous works by our group [40], the present study performed a numerical investigation for a jointed rock slope in the light of the geological conditions of a case study. An integrated approach using discrete fracture network and based on the improved hybrid FEM was proposed. In section 2, computer algorithms were proposed to generate a DFN and to deal with the grid problem for the study site. Combining with SRM, the improved hybrid FEM was presented in section 3. Then, numerical simulation was carried out to study slope stability, and different methods were employed to show the differences between intact and fractured rock slope.

\section{Treatment of the stochastic discrete fractures network}

To simulate the mechanical behaviors of discrete fractures networks (DFNs) including bedding planes and random fractures which exist in jointed rock slope, an improved hybrid finite element method was employed in this study. In such approach, the effects of DFNs on fractured medium stability were considered through integrated into finite element method code. It is notable that this type of method was developed by our team [40] and also has been employed in the stability analysis of underground excavation.

\subsection{Generation of the stochastic discrete fractures}

The generation of DFNs is vital important to simulation of fractured mediums. In particular, the characteristics of a DFN need to be described a prior so that it can be generated. In practice, discrete fracture distribution often treated by following a certain statistical law. In this means, there are a lot of research works on fracture networks distributions and have been widely reported in literatures $[2,4-6]$.

Based on the above disscussiones, a stochastics discrete fracture generation algorithm was developed. According to this algorithm, we implemented it by MATLAB and obtained the model. The configuration of the jointed rock slope in our study site, Nayong bank side, is illustrated in Fig. 1, which considered the effects both of certain bedding planes and random fractures on slope stability. The model range and fracture patterns were created in the light of field investigation. To avoid the boundary effects, slope model boundaries are used with an intact rock buffer zone between the fracture network and model boundary. This treatment of boundary effects also has been employed by Bisdom et al. [39].

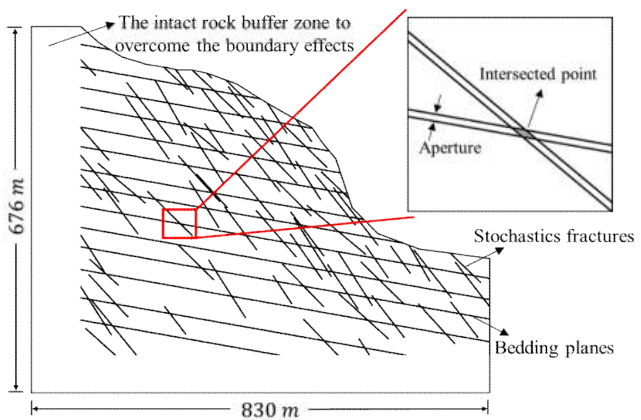

Fig. 1. Jointed rock slope numerical model considering bedding planes and stochastic fractures 


\subsection{Discretization for the jointed rock slope model}

The DFN model is vital important to the generation of finite element grids. In the situation of a complicated fracture pattern, the generation of a high-quality mesh is difficult due to the random partition configuration of the rock matrix. In addition, the discrete fractures are ubiquitous and possess a thin aperture resulting in a problem in the mesh partition process. To deal with it, a lot of literatures reported different method to carry out it. In this section, we improved a novel strategy to discrete the DFN model.

For the current model, jointed rock slope, to simulate the mechanical behavior and the aperture variation of fractures, the discrete fractures and matrix were discretized by quadrilateral elements and triangular elements, respectively. An algorithm has been developed to generate the fracture elements and the intersected elements for the discrete fractured slope model. The completed grid using fracture element generation algorithm is displayed in Fig. 2, in which the red lines indicate fracture elements.

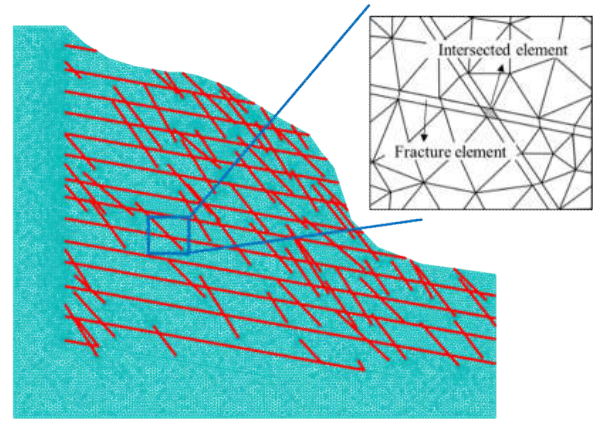

Fig. 2. Completed grid using fracture element generation algorithm and the intersected element between two fractures

\subsection{Geomechanical model for discrete fractures and rock matrix}

To describe the mechanical behaviors of stochastics fractures and bedding planes to exhibit its weakening and discontinuous effects, fracture elements were employed to deal with it. In another aspect, the rock matrix would show different response with that of fractures, thus the constitutive relations based on traditional continuum mechanics were introduced.

The fractures were modeled by the fracture elements. According to that, we have been defined the different stages for the failure conditions of fracture elements. To explain it, two key points to implement the simulation are shown as follows.

1) Elastic stage. When the normal and shear displacement, denoted by $\delta_{s}, \delta_{n}$, of fracture element are less than the critical displacement in these two directions, denoted by $\delta_{s}^{0}, \delta_{n}^{0}$, the mechanical responses of fracture element show elastic property, which are given by

$$
\left(\begin{array}{l}
\sigma_{n} \\
\sigma_{s}
\end{array}\right)=\left(\begin{array}{ll}
K_{n n} & K_{n s} \\
K_{s n} & K_{s s}
\end{array}\right)\left(\begin{array}{l}
\delta_{n} \\
\delta_{s}
\end{array}\right)
$$

Where $\sigma_{n}$ and $\sigma_{s}$ are normal and shear stress corresponding to $\delta_{n}$ and $\delta_{s}$, respectively. $K_{n n}$ and $K_{s s}$ are pure normal and shear stiffness, respectively. $K_{n s}$ and $K_{s n}$ are the coupling stiffnesses to describe the interaction between normal and shear loads, which can be neglected based on the results by [40].

2) Damage stage. In the light of above discussions, when $\delta_{n}$ and $\delta_{s}$ are greater than $\delta_{s}^{0}, \delta_{n}^{0}$, the mechanical parameters of fracture element would reduce and the effects of damage should be considered into constitutive relation.

$$
\left(\begin{array}{c}
\sigma_{n} \\
\sigma_{s}
\end{array}\right)=(1-D)\left(\begin{array}{ll}
K_{n n} & K_{n s} \\
K_{s n} & K_{s s}
\end{array}\right)\left(\begin{array}{l}
\delta_{n} \\
\delta_{s}
\end{array}\right)
$$

Where $D$ is the damage factor introduced by [54]. Based on the solving algorithm in the present work, the formula of $D$ is defined by

$$
D=\frac{\delta_{m}^{f}\left(\delta_{m}^{\max }-\delta_{m}^{0}\right)}{\delta_{m}^{\max }\left(\delta_{m}^{f}-\delta_{m}^{0}\right)}
$$

Where $\delta_{m}$ is effective displacement to describe the effects of combination of $\delta_{n}$ and $\delta_{s} \cdot \delta_{\mathrm{m}}^{\max }$ is the effective displacement threshold when the damage start. $\delta_{m}^{0}$ and $\delta_{m}^{f}$ are the effective displacement at peak and final failure of fracture element, respectively.

To decide the condition that when does the damage process occur, a criterion was used in our model, which is proposed by [41], and the peak values of stress are denoted as $\sigma_{n}^{0}$ and $\sigma_{s}^{0}$ 


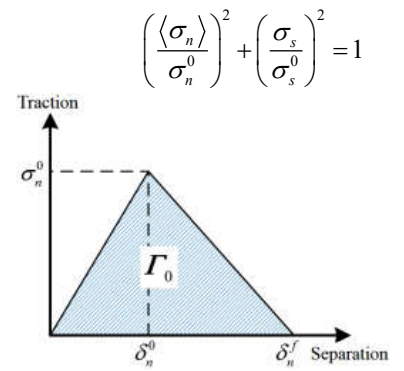

Fig. 3. Traction-separation law of fracture element during deformation

As shown in Fig. 3, the curve of fractue element during deformation is given [40], where the symbol $\Gamma_{0}$ denotes dissipated energy. The damage factor is motivated while the stress condition satisfying the above equation. Where the symbol $\left\langle\sigma_{n}\right\rangle$ indicates the $\sigma_{n}=0$ under compression condition.

\section{An improved hybrid FEM approach combining with SRM}

The above discussions render a fundamental to the hybrid finite element method improved by us. To simulate the fractured medium and to include the effects of discrete fractures on it, there are many works on this field [26-32], in which the hybrid FEM is one of the most widely used approach for the problem. Several types of the method have been proposed in the light of specific problems [30]. The general treatment of the improved method tackles the discontinuous surfaces and continuous matrix in a unified model. In our simulation, an important task is to calculate the safety factor (SF) of jointed rock slope. Based on the strength reduction method (SRM) proposed by Zienkiewicz [18], we integrated SRM into the hybrid FEM considering the impact of discrete fractures to obtain SF. The flowchart of the numerical approach for jointed rock slope is displayed in Fig. 4.

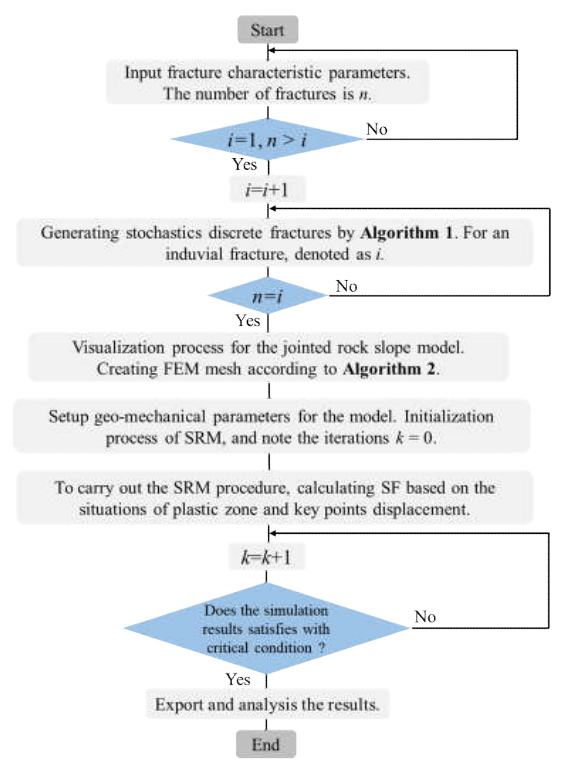

Fig. 4. Flowchart of the numerical approach for jointed rock slope

\section{Numerical results and discussions}

As mentioned above, a case study for a jointed rock slope was carried out using our improved hybrid FEM format based on the field investigation results for Nayong bank slope in Liupanshui city, Guizhou, China. The stochastic discrete fractures and bedding planes were considered by integrated the DFNs into the model. According to geological prospecting data, the overall stability condition is mainly affected by the complex fracture networks and the natural strati-graphic interfaces. In the following analysis, we focus on the mechanical behaviors of jointed rock slope under different boundary conditions, for instance, natural state and loading state. Another important intend for the simulation is to compare the differences between intact and fractured slopes to investigate the effects of discrete fractures on landslide and failure modes. 


\subsection{Geomechanical model for discrete fractures and rock matrix}

To analyze the stability condition of the jointed rock slope under a natural wording situation, we setup a boundary condition, where the model only subjected to the gravity force $\left(\mathrm{g}=9.8 \mathrm{~m} / \mathrm{s}^{2}\right)$. It is essential to carry out the stress initialization step, during which movement of fracture surfaces and model boundaries were constrained. After finished stress initialization and displacement balance procedures, the displacement state was released to let the whole model equilibrium. The mechanical parameters used for simulation is listed in Table 1.

Table 1 Mechanical properties of rock for the study site

\begin{tabular}{ccccc}
\hline Elasticity modulus & Poisson's ratio & Frictional angle & Cohesion & Density \\
\hline $10.5 \mathrm{GPa}$ & 0.25 & $42^{\circ}$ & $1.8 \mathrm{MPa}$ & $2600 \mathrm{~kg} / \mathrm{m} 3$ \\
\hline
\end{tabular}

To obtain the safety factor (SF) using the improved hybrid FEM and strength reduction method (SRM), according to the flowchart in Fig. 4, we introduced the reduce safety factor (SRF) integrated into our numerical analysis. Until emerging an obvious plastic zone, so that a slip surface and potential landslide would be induced and resulting from mechanical parameter reduction (Fig. 5b). Focusing on the slip region, the distribution of displacement vectors corresponding to the region is illustrated in Fig. 5c. To analyze the displacement evolution for the key points during slip process, several monitoring sites in Fig. 5a were selected to illustrate it. By the way of SRM procedure, mechanical parameters such as strength force and friction angle, were reduced until forming an obvious slip surface, leading to the displacement variation of key points located in slip surface exhibiting a sudden change, Fig. 6.

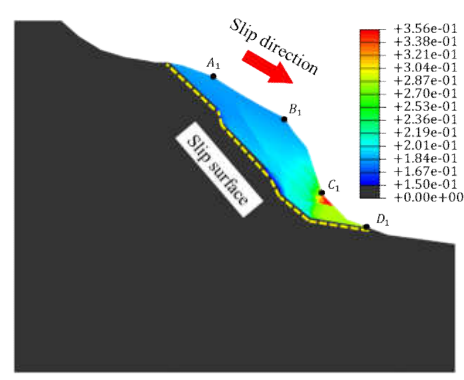

(a)

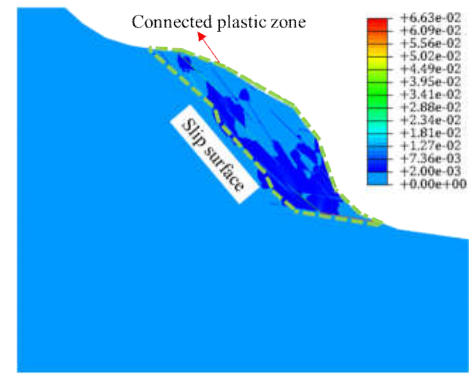

(b)

Fig. 5. Distributions of field variables in two-dimensional space when $\mathrm{SRF}=2.5$. (a) Contour of resultant displacement, where the slip surface outlines the profile according to fracture pattern (unit: $\mathrm{m}$ ). (b) Contour of equivalent plastic strain emerging a connected plastic zone caused by discrete fractures

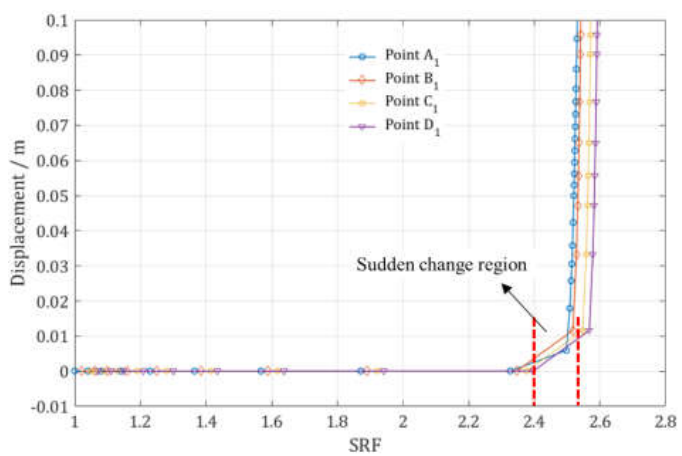

Fig. 6. Displacement evolution for key monitoring points during reduction process

\subsection{The effects of bridge foundation loading on slope stability}

It is essential to study the mechanical behaviors of the jointed rock slope during construction period, for which a large load would be applied to the slope surface induced by the bridge foundation. In the light to the bridge design plans, the foundation loads including both axial force $p_{f}=830578 \mathrm{kN}$ and bending moment $M_{f}=1250283 \mathrm{kN} \cdot \mathrm{m}$, as shown in Fig. 7. From this figure, it is obvious that bridge foundation loads lead an influence region located near the position of foundation. On the other hand, comparing with the result of displacement contour displayed in Fig. 5a, the slip surface mode in the case of loading condition is significantly different from that of natural condition. The main factor which induced this difference is loading effects. It is reasonable to imply that the bridge foundation load would lead to the reduction of SF. 


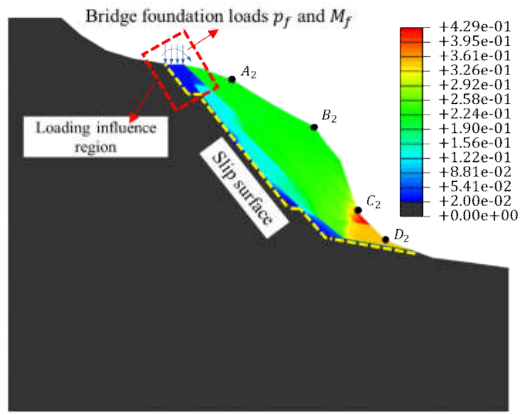

Fig. 7. Distributions of resultant displacement when $\mathrm{SRF}=2.42$. Compared with Fig. $4 \mathrm{a}$, the loading influence region indicates that the slip surface outlining effects of fracture pattern and bridge foundation loading (unit: $\mathrm{m}$ )

\subsection{Sensitivity analysis for model parameters}

To study the effects of different model parameters on the stability situations for the jointed rock slope, including fracture and rock matrix parameters, a sensitivity analysis was carried out to calculate the variation of safety factor (SF) under different mechanical parameters. Fig. 8 displays two sets of evolution curve between SF and material parameters under different conditions, which implies that both the parameters of discrete fractures and rock matrix have influence on slope stability. The parameters of friction angle and $K_{i n}$ reflect the mechanical properties of matrix and fractures, respectively. An increased magnitude of fracture parameter would induce a larger value of SF. It is obvious that the gradient variation of curves shown in Fig. 8a is smaller than that of in Fig. 8b, which also indicates the effects of fracture properties on slope stability are stronger than that of matrix. This conclusion shows the stability of a jointed rock slope is mainly controlled by discrete fractures and the attitude of stratum, where the effects of matrix property contribute a little proportion for that. [42] also has been studied the sensitivity analysis for model parameter of slope stability.

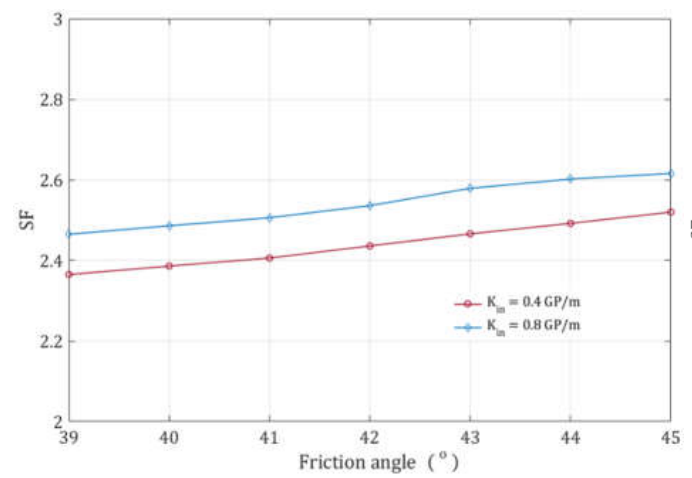

(a)

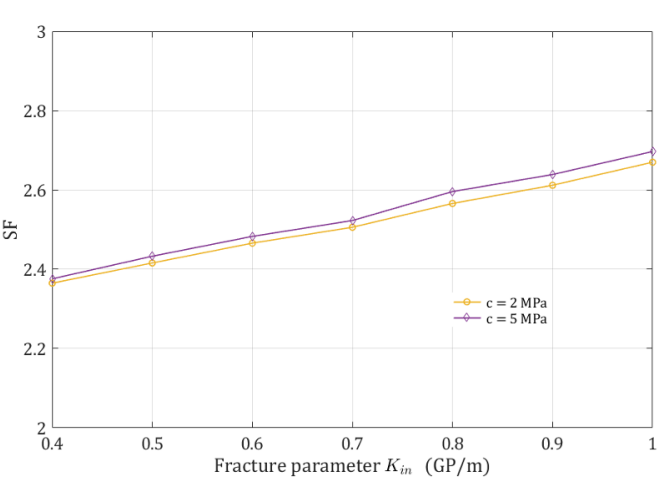

(b)

Fig. 8. Safety factor (SF) versus fracture parameter under different values of cohesion forces. (a) the effects of matrix parameter on $\mathrm{SF}$ under different fracture parameter. (b) the effects of fracture parameter on SF under different cohesion force

\subsection{Comparison analysis by different approaches: intact versus fractured rock slopes}

To analyze the influences of stochastic discrete fractures on slope stability, we carried out a comparison analysis between these two different types of slope. Corresponding to the simulation results of loading condition shown in Fig. 5, the distributions of field variables (e.g. resultant displacement and equivalent plastic strain) are displayed in Fig. 9. There is another popular method to analyze the stability of slope, limit equilibrium method (LEM) [16]. The basic hypothesis of this method is to consider slope stability as an equilibrium problem. We employed a commercial code SLIDE to implement a LEM analysis in the light of conditions for the case study, as shown in Fig. 10. By the means of sliding surface search algorithm, there are many slip surfaces and all of them are circular, in which the minimum value of SF is 2.86. In general, the value of SF calculated by LEM is often great than that of obtained by our proposed method. This difference is mainly caused by not including the contributions of fractures. Because of the approach is based on the limit equilibrium theory, as a result, it cannot integrate a discrete fracture network into analysis procedure. A lot of 
improved suggests have been proposed to revise this problem, however, it still in the framework of equivalent approach, which is oversimplify for a natural fractured rock slope.

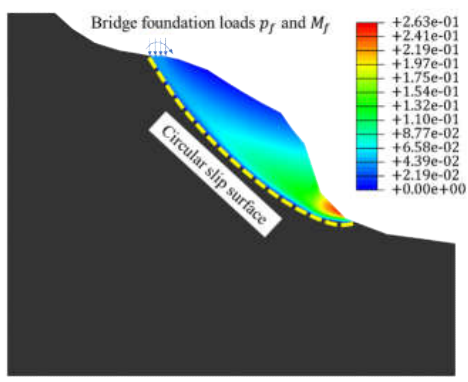

(a)

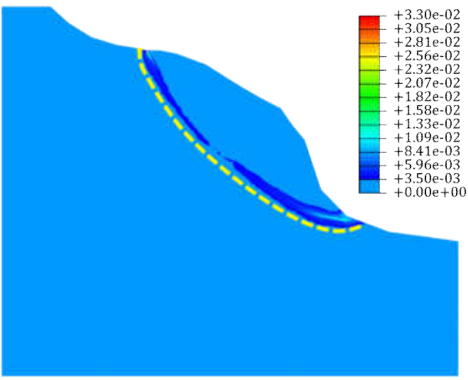

(b)

Fig. 9. Distributions of field variables under loading condition when $S R F=2.96$. (a) Contour of resultant displacement, where the slip surface is circular (unit: m). (b) Contour of equivalent plastic strain

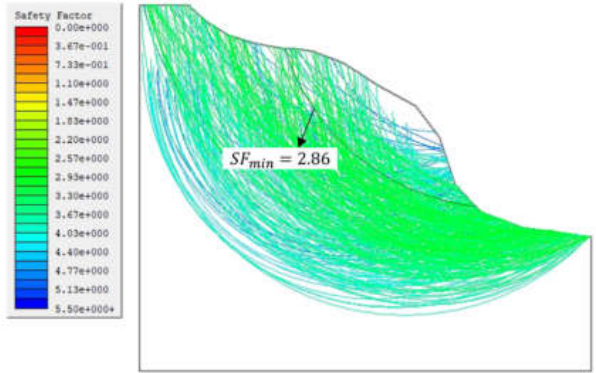

Fig. 10. Result of rock slope stability analysis by LEM analysis.fects of fracture pattern and bridge foundation loading (unit: $\mathrm{m}$ )

\section{Conclusions}

To investigate jointed rock slope stability, an integrated approach using discrete fracture network based on an improved hybrid FEM was proposed to study it. A typical jointed rock slop, Nayong bank slope, located in Liupanshui city, China, was selected as a case study and then was simulated in the light of the geological conditions for the study site. The main conclusions are presented as follows:

(1) Fracture network parameters for the study site were determined according to the data from geological survey. Based on the field investigation results, the overall stability of Nayong bank slope is mainly controlled by random fractures and bedding planes.

(2) The presented jointed rock slope model using an improved hybrid FEM, which is entirely different with the traditional model based on the framework of continuum mechanics. Thus the effects both of stochastic fractures and bedding planes were integrated into our numerical model. To analyze slope stability in a quantitative way, safety factor was calculated by the strength reduction method, and this procedure was also included in the numerical model.

(3) Numerical investigations were carried out to show the mechanical behaviors of jointed rock slope under different boundary conditions. Considering the loads induced by bridge foundation during construction period, slip surface mode in the case of loading condition is significantly different from that of in natural condition. Consequently, the impacts of bridge foundation on slope stability are reflected by the variation of slip surface patterns. It is reasonable to infer the bridge foundation would lead to a reduction of SF value.

(4) To compare the differences between intact and fractured slopes, we analyzed slope stability through different approaches. In the situation of intact rock slope, circular slip surface is the main instability mode, but for a fractured rock slope, polygonal slip surface is the major form. From the simulation results, we know that the value of SF calculated by hybrid FEM is smaller than that of by conventional FEM and LEM. This difference is mainly caused by not considering contributions of fractures.

\section{References}

1. L.R. Jing, Int..J. Rock. Mech. Min. Sci. 40, 7 (2003)

2. $\quad$ P.M. Adler, J.F. Thovert, Fractures and Fracture Networks (Netherlands, Kluwer Academic Publishers, 1999)

3. J.C. Jaeger, N.G.W. Cook, R. Zimmerman, Fundamentals of rock mechanics (Hoboken, John Wiley \& Sons, 2009)

4. B. Berkowitz, Adv. Water Resour. 25, 8 (2002)

5. W.S. Dershowitz, H.H. Einstein, Rock Mech. Rock Eng. 21, 1 (1988) 
6. Q.H. Lei, J.P. Latham, C.F. Tsang, Comput. Geotech. 85 (2017)

7. T. Belytschko, W.K. Liu, B. Moran, (Hoboken, John Wiley \& Sons, 2013)

8. R. Borst, Int. J. Numer. Methods Eng. 52, 1 (2001)

9. D.V. Griffiths, P.A. Lane, Géotechnique 49, 3 (1999)

10. E.M. Dawson, W.H. Roth, A. Dresche, Géotechnique 49, 6 (1999)

11. S. Oberhollenzer, F. Tschuchnigg, H.F. Schweiger, J. Rock Mech. Geotech. Eng. 10, 6 (2018)

12. D.Q. Li, D. Zheng, Z.J. Cao, X.S. Tang, K.K. Phoon, Eng. Geol. 203 (2016).

13. X. Fu, Q. Sheng, Y. Zhang, J. Chen, S. Zhang, Z. Zhang, Comput. Geotech. 92 (2017)

14. S. Metya, T. Mukhopadhyay, S. Adhikari, G. Bhattacharya, Comput. Geotech. 87 (2017)

15. T. Carlà, E. Intrier, P. Farina, N. Casagli, Int. J. Rock Mech. Min. Sci. 93 (2017)

16. J.M. Duncan, J. Geotech. Eng. 122, 7 (1996)

17. A.W. Bishop, Géotechnique 5, 1 (1955)

18. O.C. Zienkiewicz, C. Humpheson, R.W. Lewis, Géotechnique 25, 4 (1975)

19. K. Zhang, P. Cao, J. Meng, K. Li, W. Fan, Rock Mech. Rock Eng. 48, 2 (2015)

20. S. Wu S, L. Xiong, S. Zhang, Int. J. Geomech. 18, 10 (2018)

21. O.C. Zienkiewicz, G.N. Pande, Int. J. Numer. Anal. Methods Geomech. 1, 3 (1977)

22. C. Sun, J. Chai, Z. Xu, Y. Qin, X. Chen, Eng. Geol. 214 (2016)

23. M. Pirone, G. Urciuoli, Comput. Geotech. 102 (2018)

24. F. Tschuchnigg, H.F. Schweiger, S.W. Sloan, Comput. Geotech. 70, 1 (2015)

25. F. Tschuchnigg, H.F. Schweiger, S.W. Sloan, Comput. Geotech. 70, 2 (2015)

26. T. Ishida, M. Chigira, S. Hibino, Rock Mech. Rock Eng. 20, 4 (1987)

27. M.A. Brideau, D. Stead, Rock Mech. Rock Eng. 43, 3(2010)

28. G. Liu, X. Zhuang, Z. Cui, Eng. Geol. 225 (2017)

29. G. Yagoda-Biran, Y.H. Hatzor, Comput. Geotech. 71 (2016)

30. M. Barla, G. Piovano, G. Grasselli, Int. J. Geomech. 12, 6 (2011)

31. E.G. Baghini, M.M. Toufigh, V. Toufigh, Comput. Geotech. 80 (2016)

32. X.P. Zhou, Y. Zhao, Q.H. Qian, Eng. Geol. 192 (2015)

33. P. Hamdi, D. Stead, D. Elmo, J. Rock Mech. Geotech. Eng. 7, 6 (2015)

34. P. Fu, S.M. Johnson, C.R. Carrigan, Int. J. Numer. Anal. Methods Geomech. 37, 14 (2013)

35. L. Guo, X. Hu, L. Wu, X. Li, H. Ma, Int. J. Geomech. 18, 10 (2018)

36. T. Miyoshi, D. Elmo, S. Rogers, J. Rock Mech. Geotech. Eng. 10, 6 (2018)

37. . Elmouttie, G. Krähenbühl, A. Soliman, Comput. Geotech. 76 (2016)

38. J.C.S. Long, J.S. Remer, C.R. Wilson, P.A. Witherspoon, Water Resour. Res. 18 (1982)

39. K. Bisdom, H.M. Nick, G. Bertotti, Comput. Geosci. 103 (2017)

40. L.Y. Wang, W.Z. Chen, X.Y. Tan, X.J. Tan, J.P. Yang, X. Zhang, Bull. Eng. Geol. Environ. 5 (2019)

41. J.C. Brewer, P.A. Lagace, J. Compos. Mater. 22, 12 (1988)

42. S.J. Feng, Z.W. Chen, H.X Chen, Q.T. Zheng, R. Liu, Eng. Geol. 241 (2018) 